\title{
NARRATIVAS SOBRE O IMPACTO DAS TÉCNICAS DE REPRODUÇÃO ASSISTIDA NA EXPERIÊNCIA DA GESTAÇÃO
}

NARRATIVES ABOUT THE IMPACT OF ASSISTED REPRODUCTIVE TECHNOLOGIES ON THE EXPERIENCE OF PREGNANCY

\section{NARRATIVAS SOBRE EL IMPACTO DE LAS TÉCNICAS DE REPRODUCCIÓN ASISTIDA EN LA EXPERIENCIA DEL EMBARAZO}

\author{
Bruno Moraes da Silva* \\ Isabela Machado da Silva* \\ Eduardo Pandolfi Passos ${ }^{* * *}$ \\ Rita de Cássia Sobreira Lopes ${ }^{* * *}$ \\ Daniela Centenaro Levandowski****
}

\begin{abstract}
RESUMO
Este estudo teve como objetivo investigar as narrativas de mulheres que conceberam com o auxílio das técnicas de reprodução assistida (TRA), acerca do impacto do tratamento na experiência da gestação. Foram entrevistadas 24 mulheres no terceiro trimestre de uma gestação concebida com o auxílio de diferentes TRA. Os dados foram submetidos a uma análise narrativa, destacando-se temas como a maior valorização da gravidez devido ao tratamento, o medo de perder o bebê, a possibilidade de gestação gemelar, o preconceito social em relação ao uso das TRA e a tentativa de naturalização dessa experiência. Embora tenha predominado o não reconhecimento
\end{abstract}

\footnotetext{
Texto recebido em 14 de junho de 2017 e aprovado para publicação em 27 de agosto de 2018.

"Mestre em Psicologia e Saúde e graduado em Psicologia na Universidade Federal de Ciências da Saúde de Porto Alegre (UFCSPA), especialista em Saúde Coletiva pela Escola de Saúde Pública do Rio Grande do Sul, prestador de serviço da Organização PanAmericana da Saúde (OPAS/OMG Brasil), atuando no Programa Primeira Infância Melhor (PIM), na Secretaria de Saúde do Estado do Rio Grande do Sul. Endereço: Avenida Benno Mentz, 100/701-b, Porto Alegre-RS, Brasil. CEP: 91370-020. Telefone: (51) 99868-5938. E-mail: brunoms88@gmail.com.

"* Doutora e mestra em Psicologia pela Universidade Federal do Rio Grande do Sul (UFRGS), com especialização em Terapia de Família, professora no Departamento de Psicologia Escolar e do Desenvolvimento da Universidade de Brasília (UnB), professora e orientadora no Programa de Pós-Graduação em Psicologia Clínica e Cultura da UNB, psicóloga. Endereço: Instituto de Psicologia, Campus Darcy Ribeiro, Bloco a, sala a1 046/4, Asa Norte, Brasília-DF, Brasil. CEP: 72910-000.

"** Doutor e Mestre em Medicina - Ginecologia pela Universidade Federal de São Paulo (UNIFESP), médico formado pela UFRGS, com livre-docência em Ginecologia pela UNIFESP, professor titular na UFRGS, vice-coordenador do Programa de Pós-Graduação em Ciências da Saúde: Ginecologia e Obstetrícia da UFRGS, pesquisador do Hospital de Clínicas de Porto Alegre, coordenador do Laboratório de Embriologia e Diferenciação Celular CPE/HCPA e chefe do Serviço de Fertilidade e Reprodução Assistida do Hospital Moinhos de Vento. Universidade Federal do Rio Grande do Sul, Programa de Pós-Graduação em Ciências da Saúde: Ginecologia e Obstetrícia, Faculdade de Medicina - Campus Saúde. Endereço: Rua Ramiro Barcelos, 2400, $2^{\circ}$ andar, Porto Alegre-RS, Brasil. CEP: 90035-003. Telefone: (51) 3359-7861. E-mail: eduardo@segir.com.br.

**** Professora titular no Instituto de Psicologia da UFRGS, pesquisadora 1B do CNPQ na área de Desenvolvimento Humano, no Período Perinatal e nos Primeiros Anos de Vida. Endereço: Rua Ramiro Barcelos, 1853, ap. 112 - Rio Branco, Porto Alegre-RS, Brasil. CEP: 90035-006. Telefone: (51) 98426-0128. E-mail: ritasobreiralopes@gmail.com.

***** Pós-Doutorado em Psicologia pela Pontifícia Universidade Católica do Rio Grande do Sul (PUC-RS), Doutora e Mestra em Psicologia pela UFRGS, professora associada no Departamento de Psicologia da UFCSPA e nos Programas de Pós-Graduação em Psicologia e Saúde e em Ciências da Saúde da mesma universidade, coordenadora do NEEDS UFCSPA (Núcleo de Estudos em Desenvolvimento e Saúde), bolsista Produtividade em Pesquisa do CNPQ, psicóloga. Endereço: UFCSPA, Departamento de Psicologia. Rua Sarmento Leite, 245, sala 207, Porto Alegre-RS, Brasil. CEP: 90050-170. Telefone: (51) 3303-8826. E-mail: danielal@ufcspa.edu.br.
} 
explícito do impacto do tratamento na experiência da gestação, as participantes demonstraram-no em nuances de suas narrativas, ao relatarem seus sentimentos e expectativas. Os resultados sugerem a importância da atuação dos profissionais de saúde mental nesse contexto.

Palavras-chave: Tecnologia reprodutiva. Infertilidade. Gestação. Maternidade. Narrativas.

\section{ABSTRACT}

The aim of this study was to investigate the narratives of women who conceived with the help of assisted reproductive technologies (ART), and the impact of this treatment on the experience of pregnancy. Twenty-four women in the third trimester of pregnancy, all subjected to different ART, were interviewed. Data were submitted and evaluated using narrative analysis. Topics such a greater appreciation of pregnancy because of the treatment, the fear of losing the baby, the possibility of having twins, the social prejudice against ART, and the attempts to naturalize this experience were highlighted. The explicit non-recognition of the treatment's impact stood out. However, the participants expressed this impact when reporting feelings and expectations in their narratives. The results suggest the importance of a mental health professional taking part in this process.

Keywords: Reproductive technology. Infertility. Pregnancy. Motherhood. Narratives.

\section{RESUMEN}

Este estudio pretende investigar las narrativas de mujeres que concibieron con el auxilio de las técnicas de reproducción asistida (ART) sobre el impacto del tratamiento en la experiencia del embarazo. Fueron entrevistadas 24 mujeres en el tercer trimestre de una gestación concebida con ayuda de diferentes ARTs. Los datos fueron sometidos a un análisis narrativo, destacándose temas como la mayor valoración del embarazo debido al tratamiento, el miedo a perder al bebé, la posibilidad de embarazo gemelar, el prejuicio social en relación con el uso de las ARTs y el intento de naturalización de esta experiencia. Aunque haya predominado el no reconocimiento explícito del impacto del tratamiento en la experiencia del embarazo, las participantes lo demostraron en los matices de sus narrativas, al relatar sus sentimientos y expectativas. Los resultados sugieren la importancia de la actuación de los profesionales de salud mental en ese contexto.

Palabras clave: Tecnología reproductiva. Infertilidad. Embarazo. Maternidad. Narrativas. 


\section{INTRODUÇÃO}

$1 \begin{aligned} & \text { infertilidade é definida pela Organização Mundial da Saúde (OMS) como } \\ & \text { a impossibilidade de alcançar a gravidez após um ano ou mais de relações } \\ & \text { sexuais regulares sem o uso de métodos contraceptivos (Zegers-Hochschild }\end{aligned}$ et al., 2009), afetando de 10 a 15\% dos casais em idade fértil (Portugal, 2008). Para casais que se deparam com uma situação de infertilidade conjugal, o desejo de ter um filho biológico tende a envolver a superação de uma série de obstáculos (Mahon, \& Cotter, 2014).

$\mathrm{Na}$ sociedade ocidental, a maternidade tende a ser considerada como inerente à feminilidade, predominando a expectativa de que os casais tenham filhos. Houve, assim, um grande investimento científico no desenvolvimento de novas tecnologias que permitissem a casais inférteis alcançarem a gravidez (Lee, 2015). Nesse contexto, ganharam espaço as chamadas técnicas de reprodução assistida (TRA), procedimentos que envolvem a manipulação de óvulos e espermatozoides humanos com o objetivo de alcançar uma gravidez (Zegers-Hochschild et al., 2009). Entre as TRA, destacam-se:
a) a inseminação artificial (IA);
b) a fertilização in vitro (FIV); e
c) a ovodoação.

Apesar do potencial dessas técnicas, tem-se, cada vez mais, reconhecido que a infertilidade não constitui uma questão puramente biomédica (Lee, 2015), mas um evento com grandes repercussóes psicológicas e sociais na vida do casal e de suas famílias. A infertilidade tem sido, assim, relacionada a uma crise na vida do casal, de forma que podem se mostrar presentes sintomas de depressão e ansiedade, bem como sentimentos de incompletude, culpa, vergonha e sofrimento (Luk, \& Loke, 2015).

Nesse contexto, as pessoas podem se mostrar dispostas a fazer o necessário para alcançarem a gravidez. As TRA podem ser vistas como a última alternativa disponível para tal (Mahon, \& Cotter, 2014). A partir de um estudo qualitativo realizado na Irlanda, com 32 casais, Mahon e Cotter sugeriram que o tratamento pode ser encarado como "um desafio final, com dedicação focada, em que todos os recursos psicológicos e biológicos são investidos para garantir um resultado positivo" (p. 165). 
Desse modo, o uso das TRA tem sido descrito como um estressor multidimensional, pois abarca, além das questôes relacionadas à experiência de infertilidade, demandas e desafios próprios do tratamento (Verhaak et al., 2001). Os casais que a elas recorrem costumam lidar com despesas elevadas, com diversos procedimentos diagnósticos invasivos, com a sensação de perda de controle sobre as próprias vidas e com repetidos ciclos de esperança e frustração, que seguem os resultados obtidos em cada etapa do tratamento (Lee, 2015; Mahon, \& Cotter, 2014, Verhaak et al., 2001). Trata-se, portanto, de um período potencialmente estressante (Smorti, \& Smorti, 2013), que tende a ocasionar um desgaste físico e psíquico.

Uma vez que grande parte dos procedimentos são voltados a seu corpo, a mulher pode vivenciar esse processo de forma particular, sentindo-se responsável pelos resultados do tratamento e culpando-se diante de possíveis insucessos (Smorti, \& Smorti, 2013). Estudos acerca do impacto das TRA sobre a saúde mental das mulheres têm sugerido a presença de níveis médios a moderados de preocupação quanto à possibilidade de gestação, a redução dos afetos positivos durante os ciclos do tratamento, a presença de ansiedade e estresse diante da espera pelos resultados, e elevados níveis de sofrimento quando não se atinge o resultado esperado (Gameiro et al., 2015).

Dada a particularidade dessas experiências, tem-se destacado a relevância de investigar como a gestação é vivenciada nesse contexto. Estudos de revisão sobre o tema (Dornelles, Silva, \& Lopes, 2011; Hammarberg, Fisher, \& Winter, 2008) sugeriram a existência de diversas semelhanças na forma como a gestação é vivenciada por casais que conceberam com o auxílio das TRA e por aqueles que conceberam naturalmente. No entanto, algumas especificidades também têm sido assinaladas.

Em uma recente revisão sistemática da literatura, Gourounti (2016) identificou que estudos com mulheres que conceberam com o auxílio das TRA têm consistentemente concordado que estas apresentariam maiores níveis de ansiedade em relação à gestação, ou seja, uma maior preocupação com a continuidade da gravidez. Essa maior preocupação identificada nas mães que conceberam via TRA pode se mostrar relacionada às maiores chances de complicaçôes presentes em gestações concebidas dessa maneira (Gourounti, 2016), assim como ao fato de essas gestações serem vistas como especialmente preciosas (De Pascalis et al., 2012; Green, Darbyshire, Adams, \& Jackson, 2015). A partir de um estudo realizado em Portugal, Rocha (2015) sugeriu que essas mulheres tendem a se mostrar mais preocupadas em serem "boas mães". De acordo com Lee (2015), o fato de terem buscado a gravidez com tanto empenho pode contribuir para que elas se sintam particularmente pressionadas a desempenharem bem seus papéis. 
Essas mulheres, no entanto, não têm se mostrado particularmente ansiosas em relação à saúde de seus filhos após o nascimento, o que pode estar relacionado à crença de que "o embrião transferido é um embrião testado e sem defeitos" (Gourounti, 2016, p. 16). Essas mulheres também demonstraram pensar mais no futuro de seus bebês, especialmente no que se refere às atividades que gostariam que fizessem juntos (Rocha, 2015), e apresentar maiores índices de apego materno-fetal (Gourounti, 2016).

Além disso, estudos têm destacado a presença de atitudes mais positivas em relação à gravidez entre as mães que conceberam via TRA (Gourounti, 2016). Essas mulheres tendem a apresentar maiores níveis de satisfação e perceberiam suas gestações como mais vulneráveis, mas também mais recompensadoras. A gravidez seria vista, nesse contexto, como um momento precioso (Mahon, \& Cotter, 2014), como já mencionado.

No que se refere aos métodos utilizados para a realização de estudos sobre este tema, destaca-se o uso de escalas padronizadas e de análises quantitativas, priorizando-se a identificação de tendências gerais e a comparação entre grupos (Dornelles et al., 2011). Embora a contribuição desses estudos seja indiscutível, é importante considerar que tais estratégias metodológicas podem dificultar a compreensão das particularidades de cada família (Belsky, \& Fearon, 2004) e o acesso a aspectos subjetivos (Leiblum, 1997), o que reforça a necessidade de que o tema seja estudado a partir de diferentes métodos.

Nesse sentido, a abordagem narrativa tem sido proposta como um recurso valioso em estudos que investigam temas relacionados à infertilidade e às TRA (Daniluk, 2001). A experiência da infertilidade e das TRA, bem como a própria transição para a parentalidade podem ser consideradas como eventos que levam os envolvidos a reavaliar sua história de vida e a atribuir-lhe novos significados (Stephens, 2011). A abordagem narrativa busca justamente analisar como as pessoas compreendem aquilo que lhes ocorreu e como relacionam passado, presente e futuro (Riessman, 2008).

Estudos narrativos voltados à investigação da experiência da gestação após a infertilidade têm demonstrado como pode ser difícil superar a infertilidade ao se constatar a gravidez. Smorti e Smorti (2013), em investigação com 15 casais italianos, sugeriram que, durante os primeiros meses de gravidez, seria especialmente difícil se distanciar da experiência da infertilidade, o que explicaria os elevados níveis de ansiedade em relação à gestação e o estado de monitoramento em que algumas dessas mães se encontrariam. Já HaCohen, Amir e Wiseman (2016) compreenderam que a gravidez após a infertilidade constitui um processo de transição identitária, ou seja, um período em que a identidade é reconstruída. 
Em estudo com 12 mulheres israelenses que conceberam após um longo período de infertilidade, esses autores revelaram três tipos de narrativas que podem ser apresentadas durante esse período:

a) aquela em que a infertilidade segue ocupando lugar de destaque e em que a ansiedade a ela relacionada persiste;

b) aquela em que há uma dissociação em relação à infertilidade, de forma que os sentimentos relacionados a essa experiência devem ser silenciados e a própria força destacada; e

c) aquela em que os sentimentos sobre a gravidez e a infertilidade se mostram integrados, recebendo cada experiência o seu espaço e reconhecimento.

Considerando a escassez de estudos sobre o tema no contexto nacional bem como a potencial contribuição do método narrativo, investigaram-se, neste estudo, as narrativas de mulheres que conceberam com o auxílio das TRA acerca do impacto do uso dessas técnicas na experiência da gestação. Mais especificamente, buscou-se responder às seguintes questóes: como as mulheres relacionam, em suas narrativas, o tratamento, a experiência da gestação e suas expectativas em relação ao seu futuro e ao futuro do bebê? O impacto das TRA sobre a experiência da gravidez é explicitamente reconhecido ou se manifesta apenas nas nuances de sua narrativa?

\section{MÉTODO}

\subsection{Participantes}

Vinte e quatro mulheres, com idades entre 25 e 44 anos, de diferentes níveis socioeconômicos, que coabitavam com seus companheiros, no Estado do Rio Grande do Sul. Entre elas, $84 \%$ alcançou a gravidez a partir da FIV, enquanto o restante fez uso da IA (8\%) ou da ovodoação (8\%). A causa da infertilidade foi descrita por elas como feminina (67\%), masculina (17\%), indeterminada (12\%) ou mista (4\%). As participantes encontravam-se no terceiro trimestre de gestação, sendo que $75 \%$ delas esperavam apenas um bebê, enquanto $25 \%$ esperavam gêmeos, trigêmeos ou quadrigêmeos.

As participantes foram selecionadas entre os casais que participaram do projeto, intitulado Repassi (Lopes, Piccinini, Dornelles, Silva, \& Passos, 2007). 
Esse projeto teve como objetivo investigar a transição para a parentalidade e a relação conjugal no contexto da reprodução assistida, desde a gestação até o primeiro ano de vida do bebê, e contou com a participação de casais indicados pela equipe do Serviço de Ginecologia e Obstetrícia de um hospital público da cidade de Porto Alegre. Foram inclusas neste estudo todas as participantes que completaram a primeira fase do projeto, no terceiro trimestre da gestação, com exceção de uma, que foi excluída por apresentar problemas de saúde.

\subsection{Delineamento, procedimentos e instrumentos}

Este estudo caracteriza-se como um estudo narrativo (Polkinghorne, 1995). Os dados foram analisados qualiquantitativamente. As participantes foram contatadas pelos pesquisadores por telefone, no terceiro trimestre da gestação, quando foram informadas sobre a realização do projeto. Entre os casais contatados, $26 \%$ recusaram-se a colaborar, alegando como motivos a falta de tempo e de interesse, bem como a vontade de "deixar para trás" essa experiência (Silva, \& Lopes, 2011). Com aquelas que aceitaram participar, foi agendado um encontro em sua própria residência, em seu ambiente de trabalho ou nas dependências da universidade, conforme sua preferência.

Diante do aceite para a colaboração com o estudo, as participantes leram e assinaram um termo de consentimento livre e esclarecido e responderam a uma entrevista de dados sociodemográficos e a duas entrevistas semiestruturadas. A primeira investigou como foi vivenciado o período de gravidez desde o recebimento da confirmação, bem como as expectativas em relação ao futuro. A segunda investigou a forma como foi vivenciada a relação conjugal durante a gestação e a visão apresentada a respeito da história do casal, do tratamento e da gravidez. As respostas das participantes às entrevistas foram gravadas em áudio e posteriormente transcritas para análise. Outros instrumentos foram também aplicados, inclusive com os companheiros das participantes, mas os dados obtidos a partir destes não foram objeto de análise neste estudo.

\subsection{Análise de dados}

Neste trabalho, adotou-se o método de análise narrativa, proposto por Polkinghorne (1995), em que se procede à construção de uma narrativa contextualizada. O desafio é sintetizar os dados da pesquisa em uma história que forneça sentido às informações coletadas e que contribua para responder aos objetivos do estudo. 
Com o propósito de acessar as particularidades das experiências narradas pelas participantes, elaborou-se, para cada caso, uma narrativa em que os dados foram organizados temporalmente. Dado o objetivo deste estudo, foram realçados os conteúdos e significados referentes às percepçôes das mulheres acerca do impacto do uso das TRA sobre a experiência da gravidez. No processo de construção da narrativa de cada caso, foram cumpridas as seguintes etapas:

a) leitura cuidadosa e repetida das transcrições das entrevistas do caso;

b) identificação de sentimentos, padrões e contradições relevantes para a compreensão do objetivo em questão;

c) construção da narrativa pelo primeiro autor;

d) revisão das narrativas construídas por duas coautoras deste estudo.

Durante a análise, buscou-se também compreender se as participantes reconheciam explicitamente o impacto das TRA sobre a experiência da gestação ou se esse impacto se manifestava apenas de forma implícita, ao longo de sua narrativa. Avaliou-se, assim, a resposta das participantes à pergunta "Você acha que a experiência de reprodução assistida afetou a experiência da gestação ou o período da gestação?".

Após a elaboração das narrativas de cada caso, estas foram comparadas e deuse a composição de uma narrativa global, a partir de um processo de análise qualitativa, em que se buscou considerar tanto as particularidades como as similaridades existentes entre as participantes. No processo de construção da narrativa global, procurou-se organizar os dados apresentados nas narrativas individuais em uma ordem temporal, que considerasse:

a) a busca pelo tratamento como forma de alcançar a parentalidade bem como a experiência deste;

b) a forma como relacionam o tratamento à experiência da gestação; e

c) a forma como relacionam o tratamento às suas expectativas em relação ao futuro.

Essa narrativa foi construída por um dos autores e revisada por outros dois. 


\subsection{Considerações éticas}

Este estudo foi aprovado pelo Comitê de Ética em Pesquisa da UFCSPA (Protocolo no 179.275), e o projeto maior, do qual este estudo faz parte, pela Comissão de Pesquisa e Ética em Saúde do Hospital de Clínicas de Porto Alegre (Protocolo $\mathrm{n}^{\circ}$ 07-153). Os nomes das participantes, assim como quaisquer informações que possam revelar sua identidade, não foram nem serão revelados em trabalhos e demais publicações científicas derivadas desse projeto.

\section{RESULTADOS E DISCUSSÃO}

A construção da narrativa global acerca do impacto das TRA sobre a gestação abarcou três eixos de análise:

a) O tratamento e a busca pela gravidez: refere-se a trechos das narrativas das participantes associados à busca pelo tratamento como forma de alcançar a parentalidade bem como à experiência deste;

b) $\mathrm{O}$ tratamento e a gravidez: refere-se a trechos da narrativa associados à forma como as participantes relacionam o tratamento à experiência da gestação; e

c) O tratamento e o futuro: refere-se a trechos das narrativas em que as mulheres relatam a forma como relacionam o tratamento às suas expectativas em relação ao futuro.

Apresentam-se, a seguir, algumas das narrativas que se destacaram em cada eixo de análise, discutindo-as à luz da literatura.

\subsection{O tratamento e a busca pela gravidez}

As TRA apresentam-se aos casais não apenas como uma alternativa para alcançar a parentalidade, mas como uma opção caracterizada por certas vantagens, como permitir a experiência da gravidez e a manutenção do vínculo genético entre os pais, ou ao menos um deles e os filhos: "A gente fechou a ideia de fazer com doação, assim, é o óvulo, isso não é uma adoção, com a vantagem de que eu tô gestando, com a vantagem do [marido] poder ter um filho pela via genética, né, enfim" (G9).

A ovodoação, por exemplo, pode se assemelhar a uma experiência de adoção, com a diferença de que os pais têm a oportunidade de vivenciar a gravidez e 
desenvolver uma relação com o bebê desde o seu nascimento (Golombok et al., 1996).

Assim, a gestação via TRA pode ser considerada como uma maneira "mais especial" de alcançar a maternidade: "Mas como [...] como foi uma fertilização, acho que é mais especial, né? Já tinha perdido a esperança de ser mãe, né" (G18). Esse pensamento de que a gravidez via TRA é mais valorizada, desejada e especial é corroborado por estudos quantitativos que compararam gestantes que engravidaram naturalmente e aquelas que recorreram às TRA (Gourounti, 2016).

Simultaneamente, porém, surgem questôes que podem influenciar a forma como essas mulheres se relacionam com a experiência do tratamento, tal como a crença de que a sociedade não considera normal uma gravidez concebida via TRA: "Tem pessoas que procuram esses médicos e que negam até a morte pra qualquer um: 'Não, eu não fiz tratamento nenhum, engravidei normal'. Pra mim, não é vergonha nenhuma" (G7). Hjelmstedt, Widtröm, Wramsby e Collins (2004), em uma pesquisa realizada na Suíça, sugeriram que a preferência pela revelação do tratamento mostrou-se relacionada à visão de que sua realização constituiria uma evidência a respeito do quanto esse filho foi desejado, bem como à percepção de que o tratamento não representaria uma vergonha e, ou, algo a ser escondido. No entanto, de acordo com a revisão da literatura realizada por Silva et al. (2015), estudos têm demonstrado que, no Brasil, a infertilidade tende a estar relacionada a preconceitos, tanto por quem se descobre infértil como pela população em geral. Por isso, mulheres que se submeteram ao uso de TRA podem se refugiar no ocultamento (Straube, 2007). Também se pode pensar que a escolha por não contar sobre a realização do tratamento pode estar relacionada ao impacto gerado pela infertilidade sobre a autoestima dessas gestantes (Ribeiro, 2006).

Além das questôes sociais, as mulheres que recorrem às TRA precisam enfrentar um processo marcado por medicalização e intervençôes, por vezes, invasivas (Lee, 2015; Mahon, \& Cotter, 2014, Verhaak et al., 2001), assim como as incertezas que o acompanham: "Eu acho que ela é diferente duma gestação normal [...] Primeiro porque tu começa toda uma preparação antes, né, começa com medicação. Tu toma bastante medicação [...] E tu tem toda aquela expectativa assim, vai dar ou não vai dar, sabe” (G15).

Nesse contexto, as dúvidas sobre se o casal alcançará ou não a concretização da gravidez podem se sobressair, caracterizando-se um estado de desesperança em relação ao tratamento: "Não acreditava que ia dar, tem tanta gente que está tentando três, quatro vezes e não conseguiu" (G6). 
Uma das estratégias médicas utilizadas como forma de favorecer o sucesso do tratamento refere-se ao uso, em algumas das TRA, da implantação de múltiplos embriōes, o que também se mostra potencialmente relacionado à forma como essas mulheres vivenciam o tratamento: "E aí coloquei três embriões [na FIV], que isso foi uma coisa que nos deixou muito nervosos, porque nós não queríamos mais de um filho, né [...] Então, era um medo muito grande" (G10). Ribeiro (2014) destacou como a implantação de múltiplos embrióes em uma FIV pode dar origem a fantasias relacionadas a uma possível gravidez múltipla, sendo que as mulheres que passaram por essa experiência tendem a se dividir entre aquelas que temem essa possibilidade e aquelas que a desejam para não terem de se submeter a novos tratamentos no futuro.

\subsection{O tratamento e a gravidez}

Receber a notícia do sucesso do tratamento e, consequentemente, da gravidez mostra-se como um momento marcado tanto por medos como pela felicidade decorrente de uma sensação de vitória: "Uma gestação que tu procura reprodução assistida é porque tu já tentou a gestação normal e tu não conseguiu, então é uma coisa, assim, tipo é um troféu pra ti. Pra mim, foi o maior troféu da minha vida!" (G7). Retoma-se, assim, a ideia de que o tratamento faria com que a gravidez fosse mais valorizada ou de que, para mulheres que concebem via TRA, as dificuldades da gestação se tornam secundárias ante a satisfação de conceber (Gourounti, 2016; McMahon, Tennant, Ungerer, \& Saunders, 1999): "De repente, se eu não tivesse tido toda a função que foi, eu acho que, de repente, eu não daria tanto valor para a gravidez" (G2); "Como era uma alegria muito esperada, tudo superou a dor, qualquer coisa assim. Então, eu acho que essa alegria supera qualquer coisa" (G5).

Essa percepção de uma gravidez mais valorizada e delicada, bem como todo o desgaste vivenciado durante o tratamento (e o receio de ter que passar novamente por essa experiência) podem se mostrar relacionados ao medo de que algo venha a acontecer com a gravidez ou o bebê (De Pascalis et al., 2012; Gourounti, 2016; Smorti, \& Smorti, 2013): "Aí, depois, a outra preocupação era manter até o terceiro mês, porque a doutora tinha dito que, às vezes, tem que ter um certo cuidado, aquela coisa toda, né?" (G2); "Eu me cuido muito mais, até por ser uma fertilização, que eu acho assim que [...] muito mais delicado. Então, eu acho que todo o cuidado que eu tenho hoje é por saber que foi feita uma fertilização" (G22).

Os temores em relação a uma possível gestação gemelar vêm a se concretizar para algumas das mulheres que recorrem às TRA. Nesses casos, as fantasias dão 
lugar à necessidade de ter que se preparar para a chegada de mais de um bebê, em um contexto em que coexistem medo e felicidade (Ribeiro, 2014): "Foi um choque, assim [ao receber a notícia de que eram gêmeos], né, porque, ao mesmo tempo que a gente queria, e quer muito, assim, veio aquele negócio, assim, a gente ficou meio desbaratinado" (G15).

Aliando-se a esses medos a ideia de que a sociedade pode apresentar dificuldades em ver a gravidez concebida via TRA como "normal", uma possível estratégia de defesa ante o estigma da infertilidade consiste na tentativa de naturalização (Straube, 2007): "Eu sempre levei, apesar de todo mundo dizer que não era uma gravidez normal. Para mim, sempre foi uma coisa normal. Eu nunca encarei como se fosse uma coisa diferente" (G5); "Antigamente, tinha aquela coisa, né, um nenê diferente, mas, assim, pra mim não, né, porque foi usado tanto dele quanto meu material, né, foi feito bem dizer [...] Só foi colocado de forma diferente, né, mas, pra mim, bem tranquilo" (G8).

Ao longo das entrevistas, as participantes responderam à questão: "Você acha que a experiência de reprodução assistida afetou a experiência da gestação ou o período da gestação?”. Das 24 entrevistadas, 6 delas (G2, G7, G15, G18 e G22) responderam afirmativamente à pergunta, ou seja, reconheceram explicitamente a existência de um impacto das TRA sobre a experiência da gravidez. Embora as demais participantes tenham respondido negativamente a essa questão, identificou-se, em outros momentos de suas narrativas, que elas relacionavam o tratamento à forma como vivenciavam sua gestação. Nesses casos, pode-se dizer que o reconhecimento do impacto das TRA sobre a gestação se deu de forma implícita.

É possível considerar que um dos motivos relacionados a essa preponderância de um não reconhecimento explícito do impacto das TRA sobre a gestação esteja relacionado à ideia de que a pergunta apresentada implicaria uma percepção negativa acerca do tratamento, o que pareceu levar as participantes a negarem a percepção de qualquer influência: "Eu não encaro nada, assim, como negativo. Para mim, eu acho que a oportunidade que eles dão é única e muito boa; não tem o que dizer, assim, de negativo" (G6).

Mesmo entre as participantes que reconheceram explicitamente o impacto do tratamento sobre a experiência da gestação, duas (G2 e G7) fizeram-no apenas em relação a aspectos que poderiam ser considerados como positivos, ou seja, a maior valorização da gestação. No entanto, no decorrer de suas narrativas, foi possível identificar também temores e receios relacionados ao tratamento. Assim, aspectos, como a questão da gemelaridade e o medo de que algo pudesse 
acontecer ao bebê só foram manifestados em nuances de suas narrativas e não quando perguntadas diretamente sobre o tema.

Conforme HaCoen, Amir e Wiseman (2016) revelaram em seu estudo, realizar a transição entre a infertilidade e a gravidez pode se mostrar desafiador, de forma que algumas gestantes tendem a se distanciar de uma ou de outra experiência. No caso deste estudo, é possível supor que essas gestantes ainda estejam elaborando todo o processo por que passaram, de forma que reforçar os aspectos "positivos" da experiência do tratamento seria uma forma de lidar com essa possível ambivalência, principalmente por estarem vivendo um momento que se supõe ser de realização e alegria.

Outra possibilidade se refere a essas mulheres acreditarem que não podem se queixar após finalmente terem engravidado. Harf-Kashdaei e Kaitz (2007), ao entrevistarem e aplicarem questionários em 30 mulheres que engravidaram via TRA e em 30 que haviam engravidado naturalmente, concluíram que o primeiro grupo mostrou-se menos suscetível a reclamar ou fazer algum apontamento sobre a gravidez em razão do sentimento de que deveriam se mostrar gratas por terem conseguido engravidar, após todas as dificuldades enfrentadas. $\mathrm{Na}$ mesma direção, Fisher, Hammarberg e Baker (2008), e Hjelmstedt et al. (2004) sugerem que mulheres que recorrem às TRA podem experimentar dificuldades em assumir que a experiência da gravidez não é marcada somente por sentimentos considerados bons. Os autores levantaram a possibilidade de essas mulheres não se sentirem no direito de reclamar ou expressar qualquer dúvida, incerteza ou sentimentos ambivalentes sobre a realidade da maternidade, após todo o esforço dispendido para alcançar a gravidez. Dessa forma, o impacto do tratamento parece ficar inacessível ou minimizado para as participantes diante da exigência de se mostrarem gratas ou, até mesmo, da satisfação por conseguirem conceber (McMahon et al., 1999).

A necessidade de compreensão acerca da diferença entre o reconhecimento explícito e o reconhecimento implícito do impacto das TRA sobre a experiência da gestação está relacionada ao fato de que a maioria dos estudos sobre o tema têm utilizado escalas e questionários (Dornelles, Silva, \& Lopes, 2011), os quais tendem a se basear em um reconhecimento explícito. Constatar que mais de $70 \%$ das participantes deste estudo responderam negativamente à pergunta "Você acha que a experiência de reprodução assistida afetou a experiência da gestação ou o período da gestação?", ao mesmo tempo em que revelaram esse impacto em outros pontos de suas narrativas, sugere que os pesquisadores devem atentar aos métodos de coleta de dados empregados em pesquisas junto a esse público. Conforme destaca Leiblum (1997), os estudos sobre reprodução assistida têm sido marcados por divergências entre clínicos e pesquisadores, sendo que os 
primeiros destacariam o potencial impacto exercido pelas TRA sobre os casais, enquanto os segundos apresentariam resultados contraditórios. Dessa forma, é possível conceber que o uso de instrumentos mais sensíveis, que permitam a identificação de temas mais sutis (Leiblum, 1997), contribuiria para um maior diálogo entre esses dois grupos de profissionais, além de possibilitar um retrato mais complexo acerca da experiência dessas mulheres.

\subsection{O tratamento e o futuro}

As preocupações vivenciadas durante a gravidez, assim como as especificidades da TRA utilizada, mostram-se relacionadas às expectativas dessas mulheres em relação a seu futuro e ao futuro do filho. Exemplo disso são as preocupações sobre as consequências da ausência do vínculo genético entre mãe e filho, que caracteriza a ovodoação: "Então, assim, a minha preocupação às vezes é 'Ai, e se esse nenê não tiver nada meu, sabe?', não tiver uma boca, um olho, uma coisa assim, né? [...] é uma coisa que, às vezes, passa pela minha cabeça" (G16).

Em estudo realizado no Brasil, Straehl, Lara, Sá, Reis e Rosa-e-Silva (2017) constataram que, de 69 mulheres que realizavam outros tratamentos para infertilidade, 53\% afirmaram que se recusariam a recorrer à ovodoação em virtude da ausência do vínculo genético. De acordo com Razavi et al. (2014), essa recusa pode ser relacionada a preocupações quanto a possíveis dificuldades no vínculo pais-bebê e as características a serem apresentadas pela criança, tal como assinalado por G16. No entanto, Straehl et al. (2017) sugerem, a partir de estudos anteriores, que o desejo de experienciar a gravidez, o parto e a amamentação pode contribuir para que as mulheres optem pela ovodoação.

Preocupações em relação à saúde do bebê após o nascimento foram também mencionadas: "Me preocupo muito que ela não venha saudável, né, até por conta, não sei, por ser tratamento, por ser, né, tanta medicação, tantas coisas assim" (G22). Em sua revisão da literatura, Gourounti (2016) assinala que gestantes que conceberam via TRA tendem a não se mostrar mais ansiosas em relação à saúde do bebê após o nascimento do que aquelas que conceberam naturalmente. No entanto, as narrativas das participantes deste estudo sugerem que as especificidades do tratamento, em especial no que se refere ao uso de medicação, bem como possíveis repercussões emocionais relacionadas à experiência da infertilidade (HaCohen, Amir, \& Wiseman, 2016; Lee, 2015; Luk, \& Loke, 2015; Smorti, $\&$ Smorti, 2013) podem contribuir para que algumas dessas gestantes relatem essa preocupação, o que pode ser causa de sofrimento. Trata-se, assim, de uma questão a que os profissionais de saúde que atuam junto a essa população devem estar atentos. 
Por fim, identificaram-se preocupações quanto à reação dos filhos ao saberem que foram concebidos via TRA:

Esses dias, saiu uma reportagem sobre o primeiro bebê de proveta do Brasil e do EUA, e eu guardei. Eu vou guardar isso para, de repente, um dia [...] Porque ele vai ter curiosidade, a gente vai ter que contar, né? Porque, às vezes, falam que a criança pode ficar um pouco revoltada, como fica aquela de criação, não entendeu o processo que ele passou, que a gente teve que fazer para conseguir que ele venha, aquela coisa toda. Eu espero que ele entenda tudo e veja o amor que a gente teve para que ele pudesse vir (G2).

A partir de uma revisão sistemática sobre o tema, Tallandini, Zanchetin, Gronchi e Morsan (2016) identificaram que os pais tendem a apresentar como motivos para não revelar a realização do tratamento à criança o fato de essa ser uma questão particular do casal, a dificuldade em encontrar a forma de fazê-lo e o desejo de proteger a criança. A narrativa de G2 refere outra motivação: o receio de que a revelação possa prejudicar o vínculo entre os pais e a criança. As evidências oriundas de estudos internacionais, no entanto, sugerem que a revelação tende a não se mostrar prejudicial à relação entre pais e filhos, e que famílias em que a revelação é feita tendem a apresentar relacionamentos mais abertos e harmoniosos, além de maior confiança (Tallandini et al., 2016).

\section{CONSIDERAÇÕES FINAIS}

Como bem assinalou Daniluk (2001), a construção de uma narrativa global a partir das falas dos participantes de uma pesquisa visa a "uma tentativa de capturar, de forma ligeiramente linear, a essência da sua jornada" (p. 124). Assim, a narrativa global construída neste estudo sugere que as TRA podem ser vistas como uma alternativa rumo à parentalidade, que apresenta a vantagem de permitir a manutenção do vínculo genético bem como concretizar a experiência da gravidez. Nesse sentido, pode ser considerada uma maneira especial de alcançar a maternidade. Esse caminho, no entanto, mostra-se marcado por incertezas e temores em relação ao resultado do tratamento, no que se inclui a possibilidade de uma gestação gemelar.

Particularmente no que se refere à experiência da gestação, as dificuldades vivenciadas durante o tratamento podem ser vistas como um fator que atribui um caráter especial à gravidez, tornando-a mais valorizada. Por outro lado, essa valorização e a percepção dessa gravidez como mais delicada parecem contribuir para o medo de que algo venha a acontecer com o bebê. As singularidades dessa experiência, porém, coexistem com as tentativas de naturalização dessa gravidez.

As expectativas dessas mulheres em relação ao futuro mostram-se atravessadas 
pela forma como vivenciaram o tratamento e como este influenciou a experiência da gestação. Nesse sentido, mostram-se presentes preocupações quanto à saúde dos filhos após seu nascimento, às possíveis consequências da ausência de um vínculo genético entre mãe e filho (característica da ovodoação) e à reação dos filhos ao saberem como foram concebidos.

De acordo com os resultados deste estudo, a gestação via TRA é ainda compreendida, muitas vezes, pelas gestantes e pela sociedade, como uma gestação não natural, e as mulheres que concebem com o auxílio desses tratamentos podem hesitar para reconhecer, de forma explícita, seus temores e dificuldades. Entre as participantes deste estudo, a maioria (75\%) só manifestou o impacto em nuances de suas narrativas, pois, quando diretamente questionadas, negaram perceber essa influência. Assim, as possíveis dificuldades decorrentes do uso de TRA parecem ficar minimizadas diante da satisfação de conseguir conceber após um diagnóstico de infertilidade e o sofrimento a ele associado. Entretanto, a gestação é, de modo geral e, em particular no contexto da TRA, uma experiência caracterizada por expressões de ambivalência e incerteza; não reconhecer isso pode colocar essas mulheres em situação de maior vulnerabilidade e sofrimento (Fisher, Hammarberg, \& Baker, 2008; HaCohen, Amir, \& Wiseman, 2016), o que sugere a importância de se disponibilizar um acompanhamento emocional para elas, dada a complexidade da maternidade via TRA.

Fatores como o tipo de tratamento realizado e o fato de já ter filhos se mostraram relevantes para compreender as verbalizações das participantes deste estudo. Ter outros filhos parece ter permitido às participantes terem um parâmetro com o qual comparar sua atual gestação, ao mesmo tempo em que contribuiu para o receio de ter gêmeos. Tratamentos que envolvem a doação de gametas, como a ovodoação, também mostraram exercer uma influência específica sobre a experiência da gravidez, uma vez que as consequências da falta do vínculo genético mostraram-se motivo de preocupação.

Entre as limitações deste estudo, destaca-se a abordagem retrospectiva da gestação e da experiência do tratamento, visto que as participantes, na época da entrevista, já se encontravam no terceiro trimestre gestacional. Assim, o tempo decorrido desde o término do tratamento até a confirmação e o desenrolar da gravidez pode ter permitido o distanciamento em relação a algumas experiências, colaborando para o predomínio de um não reconhecimento explícito de seu impacto.

Deve-se considerar ainda o fato de os pesquisadores terem sido apresentados às participantes pela equipe médica que realizou o tratamento de reprodução assistida. Esse fato pode ter influenciado as respostas e gerado uma preocupação 
nas participantes em passar uma imagem favorável aos pesquisadores sobre as TRA, como forma de se mostrarem gratas pela oportunidade de realização do tratamento e pelo seu sucesso (Leiblum, 1997).

De qualquer forma, este trabalho evidencia a importância de estudos qualitativos sobre esse tema, visto que a análise narrativa contribuiu para elucidar a experiência de gravidez dessas participantes. É possível supor que pesquisas quantitativas, que se baseiam em questionários diretos e dependem do reconhecimento explícito do impacto percebido, tendam a retratar um menor impacto do tratamento de reprodução assistida sobre a gestação. Dessa forma, pesquisas qualitativas podem auxiliar a elaborar novas hipóteses e ampliar a compreensão dessa realidade (Leiblum, 1997; Silva, 2013).

Reforça-se a necessidade de novos estudos nessa área, no Brasil, pois as narrativas das participantes sugerem alguns temas que podem vir a ser objeto de análise. Exemplos disso são os estilos parentais e as práticas educativas empregadas pelas mães na criação dessas crianças, nascidas após uma gravidez tão desejada e conquistada ante tantos desafios. Da mesma forma, são necessários estudos longitudinais para a avaliação do eventual impacto do uso de TRA a médio e longo prazo na vida dessas famílias, e não apenas dessas mulheres.

Espera-se que os resultados deste estudo possam ser úteis aos profissionais que trabalham com essa população. Os achados aqui obtidos reiteram a necessidade da atuação de profissionais da saúde mental para avaliar os aspectos psicológicos que podem influenciar a trajetória dessas mulheres rumo à gravidez bem como para lidar com o tratamento de reprodução assistida. A trajetória percorrida por essas mulheres mostra-se marcada por desafios que podem ser fonte de ansiedade e sofrimento, processo que pode se tornar ainda mais complexo sem uma pessoa capacitada para acolher suas demandas emocionais. 


\section{REFERÊNCIAS}

Belsky, J., \& Fearon, R. M. P. (2004). Exploring marriage-parenting typologies and their contextual antecedents and developmental sequelae. Development and Psychopathology, 16, 501-523. Recuperado a partir de https://doi.org/10.1017/ S095457940400464X

Daniluk, J. C. (2001). "If we had it to do over again...": Couples' reflections on their experiences of infertility treatment. The Family Journal, 9(2), 122-133. Recuperado a partir de https://doi.org/10.1177/1066480701092006

De Pascalis, L., Agostini, F., Monti, F., Paterlini, M., Fagandini, P., \& La Sala, G. B. (2012). A comparison of quality of life following spontaneous conception and assisted reproduction. International Journal of Gynecology and Obstetrics 118, 216-219. Recuperado a partir de https://doi.org/10.1016/j. ijgo.2012.04.020

Dornelles, L. M. N., Silva, I. M., \& Lopes, R. C. S. (2011). Gestação com o auxílio das técnicas de reprodução assistida: uma revisão da literatura. Interação em Psicologia, 15(1), 89-100. Recuperado a partir de http://dx.doi. org/10.5380/psi.v15i1.16962

Fisher, J., Hammarberg, K., \& Baker, G. H. W. (2008). Antenatal mood and fetal attachment after assisted conception. Fertility and Sterility, 89(5), 1103-1112. Recuperado a partir de https://doi.org/10.1016/j.fertnstert.2007.05.022

Gameiro, S., Boivin, J., Dancet, E., Klerk, C., Emery, M., Lewis-Jones, C., . . . Vermeulen, N. (2015). ESHRE guideline: routine psychosocial care in infertility and medically assisted reproduction - a guide for fertility staff. Human Reproduction, 30(11), 2476-2485. Recuperado a partir de https://doi. org/10.1093/humrep/dev177

Golombok, S., Brewaeys, A., Cook, R., Giavazzi, M. T., Guerra, D., Mantovani, A. ... Dexeus, S. (1996). Children: the European study of assisted reproduction families: family functioning and child development. Human Reproduction, 11(10), 2324-2331. Recuperado a partir de https://academic.oup.com/ humrep/article/11/10/2324/570059

Gourounti, K. (2016). Psychological stress and adjustment in pregnancy following assisted reproductive technology and spontaneous conception: a systematic review. Women Health, 56(1), 98-118. Recuperado a partir de https://doi.org/10.1080/03630242.2015.1074642 
Green, J., Darbyshire, P., Adams, A., \& Jackson, D. (2015). Desperately seeking parenthood: Neonatal nurses reflect on parental anguish. Journal of Clinical Nursing, 24, 1885-1894. Recuperado a partir de https://doi.org/10.1111/ jocn. 12811

HaCohen, N., Amir, D., \& Wiseman, H. (2016). Women's narratives of crisis and change: transitioning from infertility to pregnancy. Journal of Health Psychology, 23(5), 720-730. Recuperado a partir de https://doi. org/10.1177/1359105316652465

Hammarberg, K., Fisher, J. R. W., \& Wynter, K. H. (2008). Psychological and social aspects of pregnancy and early parenting after assisted conception: a systematic review. Human Reproduction Update, 14(5), 395-414. Recuperado a partir de https://doi.org/10.1093/humupd/dmn030

Harf-Kashdaei, E., \& Kaitz, M. (2007). Antenatal moods regarding self, baby, and spouse among women who conceived by in vitro fertilization. Fertility and Sterility, 87(6), 1306-1313. Recuperado a partir de https://doi.org/10.1016/j. fertnstert.2006.11.035

Hjelmstedt, A., Widström, A. M., Wramsby, H., \& Collins, A. (2004). Emotional adaptation following successful in vitro fertilization. Fertility and Sterility, 81(5), 1254-1264. Recuperado a partir de https://doi.org/10.1016/j. fertnstert.2003.09.061

Lee, E. J. (2015) Making Parents? Human reproduction and family life in contemporary society. In British Sociological Association, Human Reproduction Study Group - Annual Conference, Roehampton: University of Roehampton. Recuperado a partir de http://gala.gre.ac.uk/16274/3/16274\%20GALE_ Life_is_a_Rollercoaster_2015.pdf

Leiblum, S. R. (1997). Love, sex, and infertility: the impact of infertility on couples. In S.R. Leiblum. Infertility: Psychological Issues and counseling strategies. (pp. 149-166). New York: John Willey \& Sons.

Lopes, R. C. S., Piccinini, C. A., Dornelles, L. M. N., Silva, I. M., \& Passos, E. P. (2007). Transição para a parentalidade e a relação conjugal no contexto da reprodução assistida. (Projeto de Pesquisa). Instituto de Psicologia, Universidade Federal do Rio Grande do Sul, Porto Alegre.

Luk, B. H., \& Loke, A. Y. (2015). The impact of infertility on the psychological well-being, marital relationships, sexual relationships, and quality of life of 
couples: a systematic review. Journal of Sex \& Marital Therapy, 41(6), 610-625. Recuperado a partir de https://doi.org/10.1080/0092623X.2014.958789

Mahon, E., \& Cotter, N. (2014). Assisted reproductive technology - IVF treatment in Ireland: a study of couples with successful outcomes. Human Fertility, 17(3), 165-169. Recuperado a partir de https://doi.org/10.3109/146 47273.2014.948498

McMahon, C. A., Tennant, C., Ungerer, J., \& Saunders, D. (1999). 'Don't count your chickens': a comparative study of the experience of pregnancy after IVF conception. Journal of Reproductive and Infant Psychology, 17(4), 345-356. Recuperado a partir de https://doi.org/10.1080/02646839908404600

Polkinghorne, D. E. (1995). Narrative configuration in qualitative analysis. Qualitative Studies in Education, 8(1), 5-23. Recuperado a partir de https:// doi.org/10.1080/0951839950080103

Portugal. (2008). Direcção Geral da Saúde. Programa Nacional de Saúde Reprodutiva. Saúde reprodutiva: infertilidade. Lisboa: DGS. Recuperado a partir de http://id.bnportugal.gov.pt/bib/bibnacional/1746424

Razavi, M., Razavi, F., Erfanian, S., Akbari, M., Mohtashami Far, E., Mahadyar, M; Eatesami, V. (2014). Factors influencing the acceptance of oocyte donation as an infertility treatment in infertile couples. Journal of Jahrom University of Medical Sciences, 11, 148-148.

Ribeiro, F. S. (2014). Gravidez múltipla concebida através de reprodução assistida: buscando uma compreensão analítica desta experiência contemporânea de concepção. Saarbrücken: Núcleo de Edições Acadêmicas.

Ribeiro, M. (2006). Articulações entre narcisismo e reprodução assistida. In R. M. M. Melamed, \& J. Quayle (Orgs.), Psicologia em reprodução assistida: experiências brasileiras. (pp. 91-103). Itatiba: Casa Psi.

Riessman, C. K. (2008). Narrative methods for the human sciences. Los Angeles: Sage.

Rocha, T. P. B. M. (2015). Vivência psicológica da gravidez após sucesso das técnicas de procriação medicamente assistida. (Dissertação de Mestrado). Programa de Pós-Graduação em Psicologia, Universidade Católica Portuguesa, Porto. Recuperado a partir de https://repositorio.ucp.pt/handle/10400.14/20495 
Silva, I. M. (2013). O desenvolvimento da relação do casal durante a transição para a parentalidade no contexto da reprodução assistida: um estudo longitudinal da gestação ao primeiro ano de vida do bebê. (Tese de Doutorado). Instituto de Psicologia, Universidade Federal do Rio Grande do Sul, Porto Alegre. Recuperado a partir de http://www.lume.ufrgs.br/handle/10183/71825

Silva, I. M., \& Lopes, R. C. S. (2011). Relação conjuga no contexto da reprodução assistida: o tratamento e a gravidez. Psicologia: Teoria e Pesquisa, 27(4), 449-457. Recuperado a partir de https://doi.org/10.1590/S0102-37722011000400008

Silva, I. M., Sonego, J. C., Ribeiro, F. S., Lopes, R. C. S., Piccinini, C. A., \& Passos, E. P. (2015). A parentalidade no contexto da reprodução assistida. In A. C. Bastos (Org.), Família, recurso pessoal e social: o caso do Brasil. (pp. 395418). Curitiba: Juruá.

Smorti, M., \& Smorti, A. (2013). Medical successes and couples' psychological problems in assisted reproduction treatment: a narrative based medicine approach. The Journal of Maternal-Fetal and Neonatal Medicine, 26(2), 169172. Recuperado a partir de https://doi.org/10.3109/14767058.2012.722728

Stephens, C. (2011). Narrative analysis in health psychology research: personal, dialogical and social stories of health. Health Psychology Review, 5(1), 62-78. Recuperado a partir de https://doi.org/10.1080/17437199.2010.543385

Straehl, J., Lara, L. A. S., Sá, M. F. S., Reis, R. M., \& Rosa-e-Silva, A. C. J. S. (2017). What do infertile women think about oocyte reception, oocyte donation, and child adoption? Revista Brasileira de Ginecologia e Obstetrícia, 39(6), 282-287. Recuperado a partir de https://dx.doi.org/10.1055/s-0037-1603742

Straube, K.M. (2007). Da família pensada à família vivida: estigma, infertilidadee as tecnologias conceptivas. (Dissertação de Mestrado). Programa de Pós-Graduação em Sociologia, Universidade Federal do Paraná, Curitiba. Recuperado a partir de https://acervodigital.ufpr.br/handle/1884/11732

Tallandini, M. A., Zanchettin, L., Gronchi, G., \& Morsan, V. (2016). Parental disclosure of assisted reproductive technology (ART) conception to their children: a systematic and meta-analytic review. Human Reproduction, 31(6), 1275-1287. Recuperado a partir de https://doi.org/10.1093/humrep/dew068

Verhaak, C. M., Smeenk, J. M. J., Eugster, A., Minnen, A., Kremer, J. A. M., \& Kraaimaat, F. W. (2001). Stress and marital satisfaction among women before and after their first cycle of in vitro fertilization and intracytoplasmic sperm 
injection. Fertility and Sterility, 76(3), 525-531. Recuperado a partir de https:// doi.org/10.1016/S0015-0282(01)01931-8

Zegers-Hochschild, F., Adamson, G. D., Mouzon, J., Ishihara, O., Mansour, R., Nygren, K., ... World Health Organization. (2009). International Committee for Monitoring Assisted Reproductive Technology (ICMART) and the World Health Organization (WHO) revised glossary of ART terminology. Humam Reproduction, 24(11), 2683-2687. Recuperado a partir de https://doi. org/10.1016/j.fertnstert.2009.09.009 